Pharmacology

\title{
Interaction of Green Tea Catechins with Breast Cancer Endocrine Treatment: A Systematic Review
}

\author{
Eugenia C. Yiannakopoulou \\ Department of Medical Laboratories, Faculty of Health and Caring Professions, Technological Educational Institute \\ of Athens, Athens, Greece
}

\section{Key Words}

Green tea catechins - Breast cancer - EGCG · Tamoxifen . Raloxifene $\cdot$ Synergistic interaction

\begin{abstract}
Recent data have shown strong chemopreventive and possibly cancer chemotherapeutic effects of green tea polyphenols and EGCG against breast cancer. This systematic review aims to synthesize data on the possible interaction of green tea catechins with breast cancer endocrine treatment. Electronic databases were searched with the appropriate search terms. Experimental trials suggest a synergistic interaction of green tea catechins with tamoxifen or raloxifene in the treatment of estrogen receptor-positive and estrogen receptor-negative breast cancer through estrogen receptordependent and -independent mechanisms. No evidence of an interaction of green tea catechins with aromatase inhibitors or fulvestrant has been reported. As green tea catechins are natural compounds with a rather favorable safety profile, the strategy of co-administrating green tea catechins with tamoxifen seems to be a rational approach in chemoprevention, adjuvant and metastatic breast cancer treatment that needs further investigation.

(c) 2014 S. Karger AG, Basel
\end{abstract}

\section{Introduction}

Catechins (flavan-3-oils) are the main flavonoids present in green tea. The 4 major catechins are (-)-epigallocatechin-3-gallate (EGCG), which represents approximately 59\% of all catechins; (-)-epigallocatechin (EGC), corresponding to approximately 19\%; (-)-epicatechin3 -gallate (ECG), standing for approximately $13.6 \%$, and (-)-epicatechin (EC), which encompasses approximately $6.4 \%$ [1]. Recent data have shown strong chemopreventive and possibly cancer chemotherapeutic effects of green tea polyphenols and EGCG against cancers of the skin, lung, breast, colon, liver, stomach, and prostate [25]. Most data concern EGCG, the most abundant catechin in green tea. An appealing strategy would be the combination of SERMS (selective estrogen receptor modulators) with green tea catechins. Although tamoxifen is used in the treatment of estrogen receptor (ER)-positive breast cancer, it is known that tamoxifen elicits proapoptotic effects in ER-negative breast cancer through the modulation of cell signaling pathways in an ER-independent manner [6]. However, these effects have been mostly reported with high concentrations of tamoxifen. The combination of tamoxifen with green tea catechins could enhance its action in ER-negative breast cancer. In addition, such a combination could allow a dosage reduction of breast endocrine treatment in ER-positive breast cancer and in breast cancer chemoprophylaxis, leading to an

\section{KARGER}

E-Mail karger@karger.com www.karger.com/pha
C 2014 S. Karger AG, Basel

0031-7012/14/0946-0245\$39.50/0
Eugenia C. Yiannakopoulou, $\mathrm{MD}, \mathrm{MSc}, \mathrm{PhD}$

Eleutheriou Benizelou 106 Kallithea

GR-17676 Athens (Greece)

E-Mail nyiannak@teiath.gr 
amelioration of the safety profile. This systematic review aims to synthesize data on the possible interaction of green tea catechins with breast endocrine treatment.

\section{Methods}

PubMed, Scopus, Google Scholar, and Science Citation Index were searched using the search terms 'EGCG', 'green tea', 'catechins', 'tamoxifen', 'raloxifene', 'aromatase inhibitors', 'anastrazole', 'letrozole', and 'SERMS'. The search covered the period from 1966 up to and including February 2013. Epidemiological, experimental or clinical trials, which investigated the interaction of green tea catechins with breast cancer endocrine treatment, were included. Only full publications were considered. There was no language restriction. The reference lists of all identified trials were checked for more relevant articles. Double publications were identified. For articles published in a non-European language, the English version was included; while the most complete report was included for articles published in European languages.

\section{Results}

Initially, 29 potentially relevant trials were identified, and after reviewing their titles and abstracts, 8 experimental trials were included.

\section{In vitro Studies}

Chisholm et al. [7] investigated if low concentrations of catechins with and without 4-hydroxytamoxifen (4OHT) would cause significant cytotoxicity in human breast cancer cells. MCF-7 (a breast carcinoma cell line showing a high level of ER-alpha expression), T47D, MDA-MB-231 and HS578T cells were incubated with EGCG, EGC or ECG (5-25 $\mu \mathrm{M})$ individually and in combination with 4-OHT for 7 days. None of the catechins were cytotoxic to T47D cells. Only EGCG $(20 \mu \mathrm{M})$ elicited cytotoxicity in MCF-7 cells. This combination treatment had no benefit for T47D and MCF-7 cells. All three catechins were significantly cytotoxic to HS578T cells at concentrations of $10 \mu \mathrm{M}$, while in this cell line, the combination with 4-OHT did not increase cytotoxicity. In MDA-MB-231 cells, EGCG $(25 \mu \mathrm{M})$ produced a greater cytotoxic effect than $4-\mathrm{OHT}(1 \mu \mathrm{M})$, and the combination of the two resulted in synergistic cytotoxicity [7].

Stuart et al. [8] investigated the role of apoptosis in the synergistic cytotoxicity elicited by $4-\mathrm{OHT}(1 \mu \mathrm{M})$ and EGCG $(25 \mu \mathrm{M})$ in MDA-MB-231 cells. They concluded that the combination of EGCG and 4-OHT results in an earlier and enhanced apoptotic response [8].
Sartippour et al. [9] observed that green tea increased the inhibitory effect of tamoxifen on the proliferation of the ER-positive MCF-7, ZR75 and T47D human breast cancer cells in vitro. In addition, this combination regimen was also more potent than either agent alone at increasing cell apoptosis [9].

Stuart et al. [10] demonstrated that a 7-day treatment with EGCG $(25 \mu \mathrm{M})$ and raloxifene $(1-5 \mu \mathrm{M})$ produced enhanced cytotoxicity toward MDA-MB-231 breast cancer cells compared to either drug alone. The earlier and enhanced induction of apoptosis was proposed as a potential mechanism for the synergistic effect, probably due to a reduced phosphorylation of the EGFR- and AKT-signaling proteins [10]. In another trial, the authors demonstrated that the combination of raloxifene with green tea catechins decreased the phosphorylation of 3 key signaling proteins (mTOR, Akt and EGFR) as well as induced the phosphorylation of SAPKs, JNK1/2 and P38. The above changes in protein phosphorylation were associated with a reduced nuclear localization of $\mathrm{p} 63$. Thus, the authors concluded that the mechanisms of synergistic interaction of green tea catechins with raloxifene were ER independent [11].

Sakata et al. [12] demonstrated that EGCG and tamoxifen exhibited dose-dependent antiproliferative effects on MCF-7 cells. The combination of EGCG with tamoxifen was more effective than either agent given alone [12].

Huang et al. [13] showed that a co-treatment with $5 \mu \mathrm{M}$ EGCG and $200 \mathrm{nM}$ tamoxifen had a synergistic effect in the inhibition of MCF-7 and AU565 cell growth, through the downregulation of the Skp2 protein, an S-phase kinase protein 2 (Skp2), component of the Skp1-cullin 1-Fbox protein (SCF) ubiquitin ligase complex, which modulates the p27 proteolysis, a key regulator of G1-to-S phase progression [13].

\section{In vivo Studies}

Scandlyn et al. [14] investigated the interaction of EGCG with tamoxifen in a xenograft model of ER-negative breast cancer. Athymic nude female mice were implanted with MDA-MB-231 cells and treated with tamoxifen, EGCG, EGCG plus tamoxifen or vehicle (controls) for 10 weeks. The tumor volume in EGCG $(25 \mathrm{mg} / \mathrm{kg})$ plus tamoxifen $(75 \mu \mathrm{g} / \mathrm{kg})$-treated mice decreased by $71 \%$ as compared with that of vehicle mice $(\mathrm{p}<0.05)$, whereas the tumor weight decreased by $80 \%$ compared with controls $(\mathrm{p}<0.01)$. Tamoxifen alone was not effective at suppressing ER-negative tumor growth, whereas EGCG had a modest effect on tumor growth. The authors suggested that the dominant mechanism for tumor suppression by the combination of green tea catechins with tamoxifen 
was the concomitant decrease in tumor protein expressions of mTOR and EGFR [14].

In animal experiments, Sartippour et al. [9] demonstrated that mice treated with both green tea and tamoxifen had the smallest MCF-7 xenograft tumor size and the highest levels of apoptosis in tumor tissue, as compared with either agent administered alone [9].

Sakata et al. [12] investigated the growth-inhibitory effect of EGCG and tamoxifen alone or in combination on preneoplastic lesions in $\mathrm{C} 3 \mathrm{H} / \mathrm{OuJ}$ mice. Animals were treated with green tea extract in drinking water $(1 \%$, $0.1 \%$ ), a tamoxifen pellet ( $10 \mathrm{mg} / \mathrm{animal}$, subcutaneously inoculated) or both agents in combination (1\% green tea extract plus $10 \mathrm{mg}$ tamoxifen). The tumor incidences were decreased in the treated groups. Even more importantly, in the group treated with green tea extract and tamoxifen, no tumors developed [12].

\section{Discussion}

Green tea has been found to block certain steps in carcinogenesis. Green tea inhibits the formation of PhIP (2-amino-1-methyl-6-phenylimidazo pyridine)-induced DNA adduct formation, which leads to a reduced tumorigenicity in animal chemoprevention studies. EGCG induces apoptosis of cancer cells through different pathways involving both the pro-oxidant and epigenetic modulation of apoptosis-related genes such as human telomerase reverse transcriptase [15]. Furthermore, green tea catechins have also been shown to reduce cell proliferation through the modulation of cell cycle progression $[13,16]$.

In addition, green tea catechins modulate multiple signaling pathways including the inhibition of nuclear factor- $\kappa \mathrm{B}$ signaling pathway; the inhibition of MAPKs and activator protein 1; the inhibition of nitric oxide synthesis; the inhibition of epidermal growth factor-mediated signal transduction pathways via the suppression of binding of EGF (epidermal growth factor) to its receptor; the inhibition of IGF-1 (insulin-like growth factor-1)mediated signaling pathways; the inhibition of cyclo-oxygenase- 2 overexpression, and the inhibition of proteasome activity [17]. The upregulation of tumor suppressor genes by EGCG has been reported. Green tea may also inhibit HER-2/neu signaling in breast cancer cells. In addition, green tea catechins have been reported to modulate cell signaling pathways associated with angiogenesis, metastasis and invasion including the inhibition of vascular endothelial growth factor as well as the inhibition of matrix metalloproteinases [17]. However, the reported

EGCG and Tamoxifen in Breast Cancer Treatment actions of green tea catechins are observed in high concentrations, which are difficult to be achieved in the clinical setting. This drawback could be overcome by designing green tea catechins with better bioavailability and/or by co-treatment combining breast cancer endocrine treatment with green tea catechins.

Indeed, the present review suggests that green tea catechins exhibit a synergistic interaction with SERMS in the treatment of ER-positive and ER-negative breast cancer cases. The co-administration of tamoxifen with green tea catechins would be an appealing strategy especially in the case of ER-negative breast cancer, where green tea catechins have been reported to re-activate ER. Indeed, in a very recently published study, Meeran et al. [18] have observed that the treatment of ER-alpha-negative breast cancer cells with green tea polyphenols led to the reactivation of ER-alpha expression [18].

The co-administration of green tea catechins with tamoxifen could also be useful in tamoxifen-resistant breast cancer cases, where green tea catechins have been reported to reverse the tamoxifen-resistant phenotype [19-21]. Furthermore, it has been shown that green tea polyphenols may inhibit the multidrug resistance P-glycoprotein activity, which is responsible for much of the resistance to chemotherapeutic drugs. Since orally administered tamoxifen is a substrate for the CYP3A-mediated metabolism and P-glycoprotein-mediated efflux in the intestine and liver, the presence of EGCG might inhibit this metabolic pathway [22].

The synergistic interaction between EGCG and tamoxifen has also been observed in the lung cancer cell line PC-9. Yet, EGCG has also been reported to interact synergistically with sulindac in the prevention of colon carcinogenesis in rats [23]. Most experimental data suggest a pharmacodynamic synergistic interaction between green tea catechins and SERMS via ER-dependent and -independent mechanisms [24]. However, a pharmacokinetic interaction between green tea catechins and tamoxifen has also been reported. Sakata et al. [12] have proposed a pharmacokinetic interaction between green tea catechins and 4-OHT. The authors suggest that, since 4-OHT and EGCG are both extensively glucuronidated, 4-OHT might inhibit the conjugation of EGCG and thus increase its cytotoxicity [12]. Supporting this hypothesis, in a pharmacokinetic analysis, Shin and Choi [25] reported that EGCG significantly increased the $\mathrm{AUC}_{0-\infty}$ and $\mathrm{C}_{\max }$ of oral tamoxifen in rats.

No evidence of an interaction between green tea catechins and aromatase inhibitors has been reported. Experimental data indicate that green tea catechins exert no 
aromatase-inhibitory activity [26]. However, Way et al. [27] have reported that black tea theaflavins inhibit aromatase activity and attenuate tamoxifen resistance in HER2/neu-transfected human breast cancer cells through tyrosine kinase suppression. In addition, modest aromatase inhibition by green tea has been reported [27].

In conclusion, experimental data indicate a synergistic interaction between green tea catechins and SERMS in the treatment of ER-positive and -negative breast cancer cases. As green tea catechins are natural compounds with a rather favorable safety profile, the strategy of co-administration of green tea catechins with tamoxifen seems to be a rational approach in the chemoprevention, adjuvant and metastatic breast cancer treatment that needs further investigation.

\section{Disclosure Statement}

The author declares no conflicts of interest.

\section{References}

1 Cabrera C, Artacho R, Gimenez R: Beneficial effects of green tea: a review. J Am Coll Nutr 2006;25:79-99.

-2 Yang CS, Wang H, Li GX, Yang Z, Guan F, Jin $\mathrm{H}$ : Cancer prevention by tea: evidence from laboratory studies. Pharmacol Res 2011;64: 113-122.

-3 Yuan JM, Sun C, Butler LM: Tea and cancer prevention: epidemiological studies. Pharmacol Res 2011;64:123-135.

4 Fon Sing M, Yang WS, Gao S, Gao J, Xiang YB: Epidemiological studies of the association between tea drinking and primary liver cancer: a meta-analysis. Eur J Cancer Prev 2011; 20:157-165.

-5 Mann CD, Neal CP, Garcea G, Manson MM, Dennison AR, Berry DP: Phytochemicals as potential chemopreventive and chemotherapeutic agents in hepatocarcinogenesis. Eur J Cancer Prev 2009;18:13-25.

6 Awad AB, Barta SL, Fink CS, Bradford PG: Beta-sitosterol enhances tamoxifen effectiveness on breast cancer cells by affecting ceramide metabolism. Mol Nutr Food Res 2008; 52:419-426

7 Chisholm K, Bray BJ, Rosengren RJ: Tamoxifen and epigallocatechin gallate are synergistically cytotoxic to MDA-MB-231 human breast cancer cells. Anticancer Drugs 2004;15: 889-897.

8 Stuart EC, Larsen L, Rosengren RJ: Potential mechanisms for the synergistic cytotoxicity elicited by 4-hydroxytamoxifen and epigallocatechin gallate in MDA-MB-231 cells. Int J Oncol 2007;30:1407-1412.

-9 Sartippour MR, Pietras R, Marquez-Garban DC, Chen HW, Heber D, Henning SM, Sartippour G, Zhang L, Lu M, Weinberg O, Rao JY, Brooks MN: The combination of green tea and tamoxifen is effective against breast cancer. Carcinogenesis 2006;27:2424-2433.

10 Stuart EC, Rosengren RJ: The combination of raloxifene and epigallocatechin gallate suppresses growth and induces apoptosis in MDA-MB-231 cells. Life Sci 2008;82:943-948.
1 Stuart EC, Jarvis RM, Rosengren RJ: In vitro mechanism of action for the cytotoxicity elicited by the combination of epigallocatechin gallate and raloxifene in MDA-MB-231 cells. Oncol Rep 2010;24:779-785.

12 Sakata M, Ikeda T, Imoto S, Jinno H, Kitagawa Y: Prevention of mammary carcinogenesis in $\mathrm{C} 3 \mathrm{H} / \mathrm{OuJ}$ mice by green tea and tamoxifen. Asian Pac J Cancer Prev 2011;12:567-571.

$\checkmark 13$ Huang HC, Way TD, Lin CL, Lin JK: EGCG stabilizes p27kip1 in E2-stimulated MCF-7 cells through down-regulation of the Skp2 protein. Endocrinology 2008;149:5972-5983.

14 Scandlyn MJ, Stuart EC, Somers-Edgar TJ, Menzies AR, Rosengren RJ: A new role for tamoxifen in oestrogen receptor-negative breast cancer when it is combined with epigallocatechin gallate. Br J Cancer 2008;99:10561063.

-15 Min NY, Kim JH, Choi JH, Liang W, Ko YJ, Rhee S, Bang H, Ham SW, Park AJ, Lee KH: Selective death of cancer cells by preferential induction of reactive oxygen species in response to (-)-epigallocatechin-3-gallate. Biochem Biophys Res Commun 2012;421:91-97.

$\checkmark 16$ Thangapazham RL, Singh AK, Sharma A, Warren J, Gaddipati JP, Maheshwari RK: Green tea polyphenols and its constituent epigallocatechin gallate inhibits proliferation of human breast cancer cells in vitro and in vivo. Cancer Lett 2007;245:232-241.

17 Beltz LA, Bayer DK, Moss AL, Simet IM: Mechanisms of cancer prevention by green and black tea polyphenols. Anticancer Agents Med Chem 2006;6:389-406.

18 Meeran SM, Patel SN, Li Y, Shukla S, Tollefsbol TO: Bioactive dietary supplements reactivate ER expression in ER-negative breast cancer cells by active chromatin modifications. PLoS One 2012;7:e37748.

$\checkmark 19$ Farabegoli F, Papi A, Orlandi M: (-)-Epigallocatechin-3-gallate down-regulates EGFR, MMP-2, MMP-9 and EMMPRIN and inhibits the invasion of MCF-7 tamoxifen-resistant cells. Biosci Rep 2011;31:99-108.
20 Farabegoli F, Papi A, Bartolini G, Ostan R, Orlandi M: (-)-Epigallocatechin-3-gallate downregulates Pg-P and BCRP in a tamoxifen resistant MCF-7 cell line. Phytomedicine 2010;17:356-362.

21 Farabegoli F, Barbi C, Lambertini E, Piva R: (-)-Epigallocatechin-3-gallate downregulates estrogen receptor alpha function in MCF-7 breast carcinoma cells. Cancer Detect Prev 2007;31:499-540.

22 Mei Y, Qian F, Wei D, Liu J: Reversal of cancer multidrug resistance by green tea polyphenols. J Pharm Pharmacol 2004;56:1307-1314.

23 Fujiki H, Suganuma M, Kurusu M, Okabe S, Imayoshi Y, Taniguchi S, Yoshida T: New TNF-alpha releasing inhibitors as cancer preventive agents from traditional herbal medicine and combination cancer prevention study with EGCG and sulindac or tamoxifen. Mutat Res 2003;523-524:119-125.

24 De Amicis F, Russo A, Avena P, Santoro M, Vivacqua A, Bonofiglio D, Mauro L, Aquila S, Tramontano D, Fuqua SA, Andò S: In vitro mechanism for downregulation of ER- $\alpha$ expression by epigallocatechin gallate in $\mathrm{ER}+$ / PR+ human breast cancer cells. Mol Nutr Food Res 2013;57:840-853.

25 Shin SC, Choi JS: Effects of epigallocatechin gallate on the oral bioavailability and pharmacokinetics of tamoxifen and its main metabolite, 4-hydroxytamoxifen, in rats. Anticancer Drugs 2009;20:584-588.

26 Satoh K, Sakamoto Y, Ogata A, Nagai F, Mikuriya $\mathrm{H}$, Numazawa $\mathrm{M}$, Yamada K, Aoki N: Inhibition of aromatase activity by green tea extract catechins and their endocrinological effects of oral administration in rats. Food Chem Toxicol 2002;40:925-933.

27 Way TD, Lee HH, Kao MC, Lin JK: Black tea polyphenol theaflavins inhibit aromatase activity and attenuate tamoxifen resistance in HER2/neu-transfected human breast cancer cells through tyrosine kinase suppression. Eur J Cancer 2004;40:2165-2174. 